\title{
High-throughput sequencing analysis of intestinal flora changes in ESRD and CKD patients
}

\author{
Jianguang $\mathrm{Hu}^{1,2}$, Xiaoshi Zhong ${ }^{1,2}$, Jing Yan ${ }^{1,2}$, Daoyuan Zhou ${ }^{1,2}$, Danping Qin ${ }^{1,2}$, Xiao Xiao ${ }^{1,2}$,
} Yuanyuan Zheng ${ }^{1,2}$ and Yan Liu ${ }^{1,2^{*}}$ (D)

\begin{abstract}
Background: Chronic kidney disease (CKD) disease affects gut flora by causing dysbiosis and lead to systemic inflammatory conditions. Here, we provide intestinal flora changes of CKD patients undertook different hemodialysis therapy.

Methods: From 2017 to 2019, a total of 166 patients from Guangzhou Red Cross Hospital were recruited and divided into four groups with 17 cases in healthy control group, 47 cases in CKD non-dialysis group, 49 cases in HD group, and 53 cases in PD group. Intestinal flora genome 165 rDNA sequencing and further bio-informatic analysis were performed.

Results: Decreased diversity and altered communities of intestinal flora in PD patients, in which microbial diversity was positive correlated with the albumin level were observed. A total of 20 intestinal flora phyla were detected in 166 fecal samples, divided into 3 dominant intestinal types including Bacteroides-dominant gut type, Firmicutesdominant type and Proteobacteria-dominant gut type. Further analyses found 198 genera, the abundance of 86 genera were significantly different. Butyrate-producing taxa as Faecalibacterium in genera level and Bifidobacteriaceae and Prevotellaceae in family level were dominant genus in CT, CKD, and HD groups, while urease containing-, indole- and p-cresol-forming taxa as Escherichia in genera and Enterobacteriaceae, Enterococcaceae in family level was dominated genus in PD group. Number of differential expressed genes in KEGG enrichment pathways were significantly different in PD group in carbohydrate metabolism, amino acid metabolism, energy metabolism, translation, and membrane transport.
\end{abstract}

Conclusion: Our results suggest peritoneal dialysis therapy could result in reduced diversity and altered microbial communities, with reduced probiotic butyrate-producing taxa and increased urease containing-, indole- and pcresol-forming taxa. The disordered intestinal flora can seriously affect the nutrition level in CKD patients with PD therapy.

Keywords: High-throughput sequencing, Intestinal flora, Hemodialysis, End-stage renal disease, Chronic kidney disease

\footnotetext{
* Correspondence: rabbityan127@163.com

${ }^{1}$ Department of Nephrology, Guangzhou Red Cross Hospital, Medical

College, Jinan University, Guangzhou 510220, China

${ }^{2}$ Guangzhou Institute of Disease-Oriented Nutritional Research, Guangzhou

510220, China
}

(c) The Author(s). 2020 Open Access This article is distributed under the terms of the Creative Commons Attribution 4.0 International License (http://creativecommons.org/licenses/by/4.0/), which permits unrestricted use, distribution, and reproduction in any medium, provided you give appropriate credit to the original author(s) and the source, provide a link to the Creative Commons license, and indicate if changes were made. The Creative Commons Public Domain Dedication waiver (http://creativecommons.org/publicdomain/zero/1.0/) applies to the data made available in this article, unless otherwise stated. 


\section{Background}

The human microbiome is comprised of about 100 trillion microbial cells and their encoded genes [1]. The composition of the gut microbiome varies among individuals and remains stable under homeostasis [2,3]. The most abundant bacterial phyla in a healthy human gut are Bacteroides, Firmicutes, Actinobacteria, and Proteobacteria [4]. Genetic and environmental factors, including disease, diet and antibiotic use, alter the type and abundance of the microbiome [2]. This alteration, also known as dysbiosis, causes individuals to become more susceptible to disease [5]. Hormones such as serotonin, dopamine, and norepinephrine, and microbial metabolites including p-cresol sulfate, decyloxysulfate, trimethylamine $\mathrm{N}$ oxide (TMAO), and short-chain fatty acids (SCFAs) secreted by gut microbiota, can influence various bodily functions $[6$, 7]. Kidney disease, obesity, metabolic syndrome, cancer, and cirrhosis were reported to be associated with changed endogenous flora [8-10]. So far, thorough investigations into gut-microbial-metabolite relationships under disease progression remain unclear.

Uncontrolled metabolic disorders including CKD, affect gut flora, promote intestinal permeability, cause dysbiosis, and can lead to systemic inflammatory conditions [11]. Reduced abundance of Lactobacillaceae and Prevotellaceae in CKD patients has been reported, while Enterobacter and Enterococcus were observed to be 100 times higher [12]. The secretion of uremic toxins is closely related to microbial changes in CKD patients [13]. Intestinal microbiota were associated with inflammatory status and renal function in endstage renal disease (ESRD) patients in southern China, with a decreased proportion of bacteria, and altered intestinal flora from Prevotella to Bacteroides [14]. CKD animal models have shown excessive uremia can result in intestinal dysbiosis, intestinal barrier dysfunction, and bacterial translocation [15]. Intestinal bacterial changes were found in both dialysis and non-dialysis CKD patients. Further investigations indicated that the abundance of Firmicutes and Actinobacteria in peritoneal dialysis patients was reduced, and the abundance of Bacteroides in hemodialysis patients increased [16]. However, the influence of different renal replacement therapies on microbiota remains unclear.

In this study, we examined changes in the intestinal flora of CKD patients by comparing differences in abundance, diversity, and species composition between healthy humans, CKD non-dialysis patients, HD patients, and $\mathrm{PD}$ patients, providing evidence for personalized treatment for CKD patients.

\section{Methods}

\section{Patient selection}

From 2017 to 2019, patients from Guangzhou Red Cross Hospital were recruited and divided into four groups: healthy control group, CKD non-dialysis group, HD group, and PD group. The written informed consent was signed by all patients. This study was approved by ethics committee of the Guangzhou Red Cross Hospital [No. 2017-032-01/02]. The study adhered to the tenets of the Declaration of Helsinki and the Guidance on Sample Collection of Human Genetic Diseases by the Ministry of Public Health of China. The inclusion criteria for patients in the experiment were as follows: 1) receive normal diet; 2) capable of self-care; 3) no antibiotics, antitumor drugs, immunosuppressants, and glucocorticoids over the past 3 months. The exclusion criteria for patients included the following: 1) those who cannot eat or use intestinal and/or external nutrition interventions; 2) digestive diseases including gastrointestinal cancer, biliary tract inflammation, and inflammatory bowel disease; 3) metabolic diseases including obesity, diabetes, and systemic lupus erythematosus; 4) those who with local inflammation, systemic infections before treatment. Basic clinical information of all patients was recorded. Posterior feces samples were collected and stored at $80^{\circ} \mathrm{C}$ degrees for further analysis.

\section{Intestinal flora genome $16 \mathrm{~S}$ rDNA sequencing}

Total DNA of feces was extracted using a DP328 DNA extraction kit according to the manufacturer's manual (Tiangen, Beijing, China). The total extracted genomic DNA was qualitatively detected by $1 \%$ agarose gel electrophoresis, and the concentration was determined using a Qubit ${ }^{\circ}$ dsDNA HS Assay Kit. For the $16 \mathrm{~S}$ rDNA V3 region, an upstream primer $338 \mathrm{~F}$ and a downstream primer $534 \mathrm{R}$ were used for amplification and sequencing on Illumina HiSeq2500 platform (Novogene, Beijing, China).

\section{S rDNA sequence intestinal flora analysis}

The OTU was compared using RDP classifer (v 2.2). Greengene database was used for $16 \mathrm{~S}$ bacteria and archaea genome comparison. Sliver database was used for $18 \mathrm{~S}$ fungus and UNITE database was used for ITS fungus. The Observed Species, Chao 1 index, Ace index, Shannon index, Simpson index, and Good's coverage was selected to reflect the Alpha diversity of the samples. PICRUSt was used to perform three-level Kyoto Encyclopedia of Genes and Genomes (KEGG) pathway and abundance analysis based on different numbers of $16 \mathrm{~S}$ rRNA copy numbers.

\section{Statistical analyses}

The quantitative data were expressed as mean \pm standard deviation. The mean of the two groups was compared by student's t-test. The mean of multiple groups was compared by ONE-WAY ANOVA analysis. The chi-square test was used to compare the rates between groups. Principal co-ordinates analysis was used to analyze the differences in beta diversity among different groups. The dominant intestinal type was analyzed by Multidimensional cluster analysis and Principal Component 
Analysis. All statistical analyses were performed using SPSS v22.0 (SPSS Inc., Chicago, IL, USA), with $p<0.05$ set as the difference test level.

\section{Results}

\section{Basic clinical characteristics}

A total of 166 patients were enrolled in the study, with 17 in CKD group, 47 in CT group, 49 in HD group, and 53 in PD group. No significant differences in age, gender, and body weight were found among the four groups $(p>0.05)$. Renal function tests, including uric acid, blood urea, nitrogen, and serum creatinine in the healthy control group were significantly lower than those in the other three groups $(p<0.001)$. Metabolic status, including the expression levels of albumin and TC, were significantly higher in CT group $(p<0.001)$, while FBG showed no difference among groups $(p=0.29)$ (Table 1). Primary kidney diseases were altered among three groups of CKD patients, cases of chronic nephritis in CKD, HD, and PD was 4(23.5\%), 16 (32.7\%) and $21(39.6 \%)$, the number of hypertensive nephropathy in CKD, HD, and PD was 10(58.8\%), 27(55.1\%) and 22 $(41.5 \%) \quad(p=0.002)$. In inflammatory conditions, CRP and IL-6 were significantly higher in CKD patients $(p<0.001)$ while WBC showed no difference among four groups $(p=0.18)$. CKD patients with PD showed a trend of longer dialysis vintage compared to those with HD (39.85 \pm 39.44 month vs. $35.35 \pm 32.33$ month, $p=0.532$ ).

Table 1 Comparison of basic clinical information of patients

\begin{tabular}{|c|c|c|c|c|c|c|c|}
\hline & $\operatorname{CKD}(N=17)$ & $\mathrm{CT}(\mathrm{N}=47)$ & $\mathrm{HD}(N=49)$ & $\mathrm{PD}(N=53)$ & Total & $F / X^{2}$ value & $p$ value \\
\hline$M: F$ & $13: 4$ & $23: 24$ & $28: 21$ & $29: 24$ & $93: 73$ & 3.91 & 0.272 \\
\hline Age & $57.29 \pm 11.13$ & $56.30 \pm 10.67$ & $59.53 \pm 10.57$ & $57.70 \pm 8.52$ & $57.80 \pm 10.03$ & 0.85 & 0.47 \\
\hline Weight (kg) & $57.70 \pm 13.97$ & $62.49 \pm 8.10$ & $58.12 \pm 11.63$ & $59.73 \pm 11.03$ & $59.83 \pm 10.88$ & 1.58 & 0.197 \\
\hline BUN (mmol/L) & $25.86 \pm 7.80$ & $4.63 \pm 0.93$ & $23.53 \pm 7.21$ & $18.63 \pm 6.61$ & $16.85 \pm 10.00$ & 100.37 & $<0.001$ \\
\hline Creatinine (umol/L) & $1022.94 \pm 349.12$ & $67.38 \pm 15.53$ & $963.14 \pm 286.98$ & $864.09 \pm 288.10$ & $684.02 \pm 464.44$ & 133.86 & $<0.001$ \\
\hline UA (umol/L) & $452.80 \pm 82.88$ & $318.39 \pm 63.24$ & $453.63 \pm 88.20$ & $431.17 \pm 89.58$ & $408.08 \pm 99.28$ & 26.99 & $<0.001$ \\
\hline Albumin (g/L) & $35.65 \pm 4.05$ & $42.13 \pm 3.33$ & $35.06 \pm 4.67$ & $32.97 \pm 4.35$ & $36.45 \pm 5.54$ & 43.55 & $<0.001$ \\
\hline $\mathrm{Hb}(\mathrm{g} / \mathrm{L})$ & $110.12 \pm 14.10$ & $136.72 \pm 11.53$ & $105.84 \pm 16.59$ & $101.11 \pm 24.59$ & $113.51 \pm 23.42$ & 36.59 & $<0.001$ \\
\hline WBC (10^9/L) & $7.00 \pm 1.96$ & $6.27 \pm 1.38$ & $7.35 \pm 2.83$ & $7.12 \pm 2.99$ & $6.94 \pm 2.50$ & 1.67 & 0.18 \\
\hline CRP & $5.29 \pm 2.67$ & $2.21 \pm 0.99$ & $5.13 \pm 2.67$ & $10.26 \pm 21.04$ & $5.96 \pm 12.36$ & 3.86 & 0.01 \\
\hline IL-6 & $6.68 \pm 2.98$ & $2.90 \pm 2.52$ & $6.61 \pm 3.32$ & $12.63 \pm 15.30$ & $7.49 \pm 9.71$ & 10.02 & $<0.001$ \\
\hline FBG & $4.93 \pm 0.66$ & $5.19 \pm 0.79$ & $5.13 \pm 0.67$ & $5.70 \pm 3.13$ & $5.31 \pm 1.87$ & 1.24 & 0.29 \\
\hline $\mathrm{HbAc}$ & $5.27 \pm 0.51$ & $4.91 \pm 0.51$ & $5.38 \pm 0.66$ & $5.30 \pm 0.94$ & $5.21 \pm 0.73$ & 3.98 & 0.009 \\
\hline TC & $4.26 \pm 4.05$ & $4.85 \pm 0.67$ & $4.35 \pm 1.00$ & $5.10 \pm 1.16$ & $4.72 \pm 1.02$ & 6.69 & $<0.001$ \\
\hline Dialyze number (3/4/5) & - & - & - & $5 / 45 / 3$ & & - & - \\
\hline Dialysis vintage (month) & - & - & $35.35 \pm 32.33$ & $39.85 \pm 39.44$ & & 0.63 & 0.532 \\
\hline \multicolumn{8}{|l|}{ Primary disease } \\
\hline Chronic nephritis & $4(23.5)$ & - & $16(32.7)$ & $21(39.6)$ & & 23.792 & 0.002 \\
\hline Hypertensive nephropathy & $10(58.8)$ & - & $27(55.1)$ & $22(41.5)$ & & & \\
\hline Others & $3(17.6)$ & - & $6(12.2)$ & $10(18.9)$ & & & \\
\hline \multicolumn{8}{|l|}{ NYHA classification } \\
\hline I & & & $20(40.8)$ & $24(45.3)$ & & 4.291 & 0.117 \\
\hline$\|$ & & & $17(34.7)$ & $24(45.3)$ & & & \\
\hline III & & & $12(24.5)$ & $5(9.4)$ & & & \\
\hline Coronary disease & & & & & & 1.051 & 0.305 \\
\hline Yes & & & $29(59.2)$ & $26(49.1)$ & & & \\
\hline No & & & $20(40.8)$ & $27(50.9)$ & & & \\
\hline Hypertension & & & & & & 0.962 & 0.327 \\
\hline Yes & & & $35(71.4)$ & $33(62.3)$ & & & \\
\hline No & & & $14(28.6)$ & $20(37.7)$ & & & \\
\hline
\end{tabular}

(CKD: chronic kidney disease; CT: control; HD: hemodialysis; PD: peritoneal dialysis; M: male; F: female; BUN: Blood Urea Nitrogen: UA: Uric Acid: Hb: Hemoglobin: WBC: White Blood Ccll: CRP: C-reactive protein: IL-6: Interleukin-6: FBG: Fasting Blood Glucose: HbAc: Glycated hemoglobin: TC: Total cholesterol) 
Comorbidities including coronary disease and hypertension showed no difference between PD and HD populations $(p=0.305$ and $p=0.327)$. No significant difference was found on distribution of NYHA classifications $(P=$ 0.117). (Table 1).

\section{Alpha-beta diversity analysis of intestinal flora}

Alpha and beta diversity analysis showed that the intestinal flora structure diversity (including Sob index, Chao index, Ace index, Shannon index, and Simpson index) and species diversity distance in PD group was significantly lower than CKD, CT, and HD groups under the same measurement depth $(p<0.001)$ (Fig. 1). Further analysis showed that the express of albumin affects the alpha diversity, patients showed with lower albumin level present lower intestinal flora structure diversity and vice visa (Fig. 2).

\section{Different relative abundance level of bacterial taxa}

A total of 20 intestinal flora phyla were detected in 166 fecal samples, namely Firmicutes, Bacteroidetes, Proteobacteria, Actinobacteria, Verrucomicrobia, Fusobacteria, Cyanobacteria, Synergistetes, Tenericumtes, TM7, Chloroflexi, Lentisphaerae, Euryarchaeota, Acidobacteria, Chlamydiae, Spirochaetes, OD1, Elusimicrobia, Parvarchaeota, and unclassified, mostly abundance in Firmicutes, Bacteroidetes, Proteobacteria, Actinobacteria, and Verrucomicrobia (Fig. 3a). All samples were divided into 3 dominant intestinal types including Bacteroidesdominant gut type, Firmicutes-dominant type and Proteobacteria-dominant gut type, which displayed significantly different distributions among the four treatment groups $(p<0.01)$. The relative abundance of bacteria within the phylum Bacteroidetes and Firmicutes were significantly decreased, while relative abundance of the phylum Proteobacteria was increased in PD group (Fig. 3b-d).

\section{The genus level composition of each group of intestinal flora}

Further analyses found 198 genera, the abundance of 86 genera were significantly different (Fig. 3b). 42 genera belong to the Bacteroidetes (7), Proteobacteria (7), Firmicutes (17), and Actinobacteria (11) phyla were significantly different in PD group, 20 genera belonging to Bacteroidetes (5), Proteobacteria (4), Fusobacteria (5), and Firmicutes (6) were significantly different in HD group, 21 genera belonging to Bacteroidetes (3), Proteobacteria (7), and Firmicutes (11) were significantly different in CT group and 3 genera in CKD group belonging to Bacteroidetes (1), Firmicutes (1), Actinobacteria (1) were significantly different (Fig. 4). 4 dominant genera among four groups were: Bacteroides, Faecalibacterium, Escherichia and Salmonella. Bacteroides and Faecalibacterium were dominant genus in CT, CKD, and HD groups, while Escherichia and Salmonella was dominated genus in PD group (Fig. 5).

\section{Relative abundance of indole and $p$-cresol producing taxa in family level}

Bifidobacteriaceae and Prevotellaceae was significantly decreased, while Enterobacteriaceae, Enterococcaceae were significantly increased in patients with PD compared with the other groups. A trend of increased level was found in the relative abundance of Verrucomicroblaceae (Fig. 6).

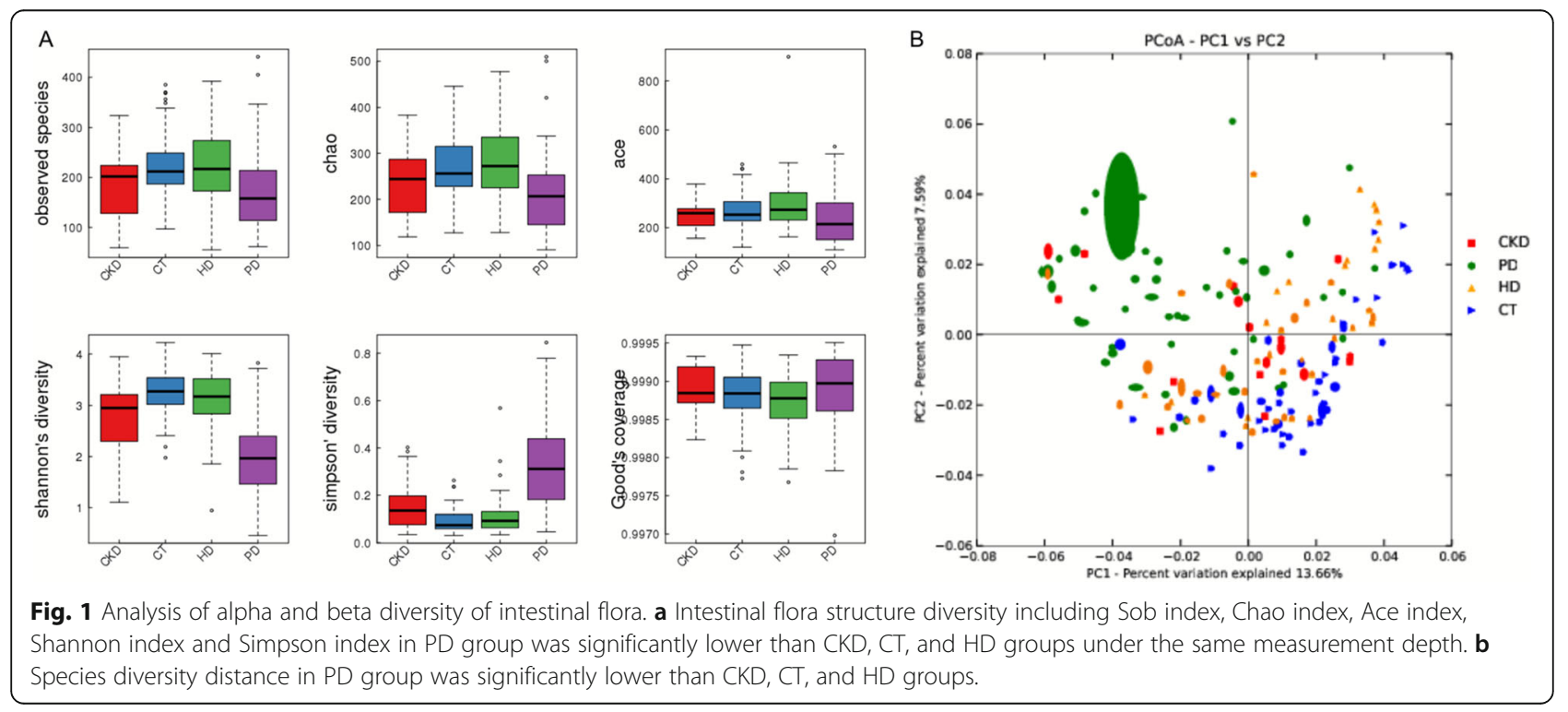




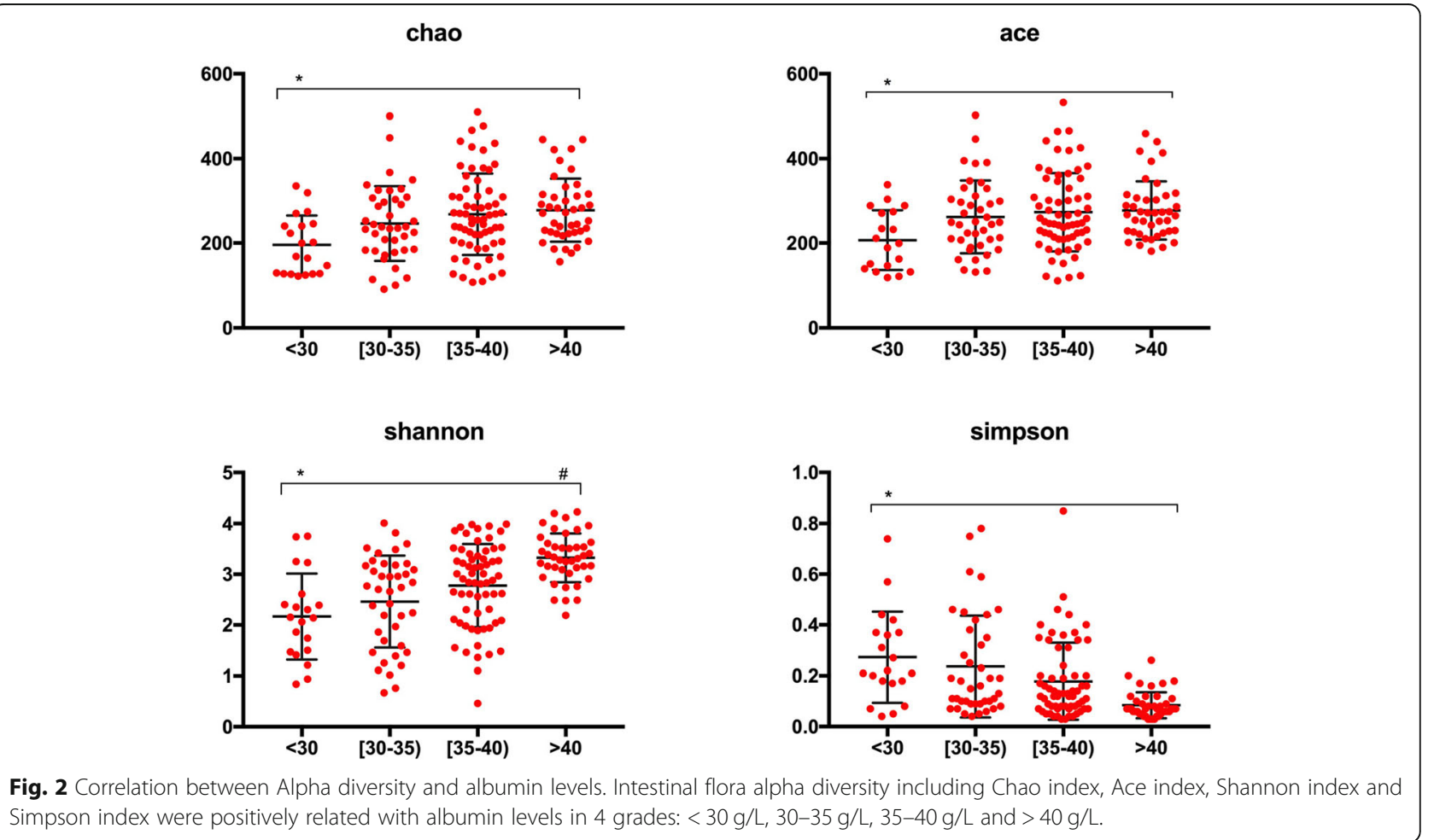

A
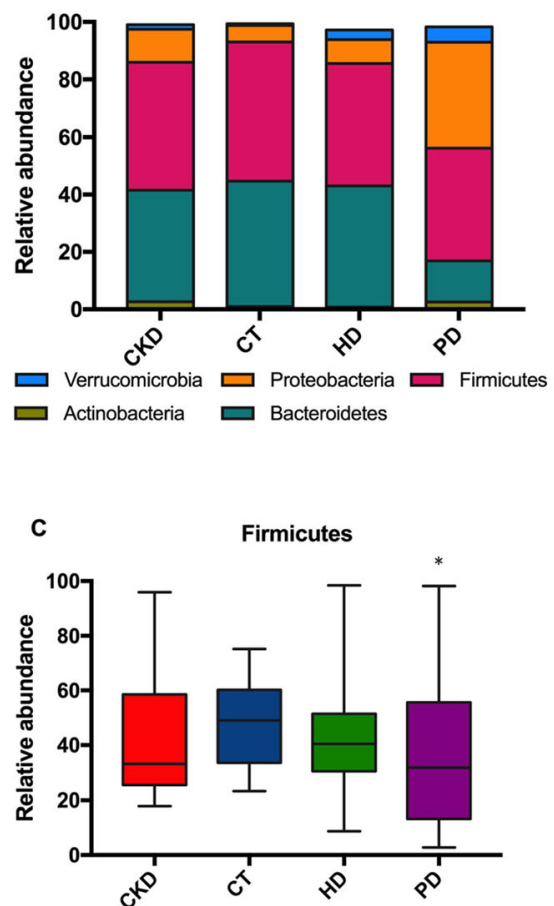

B

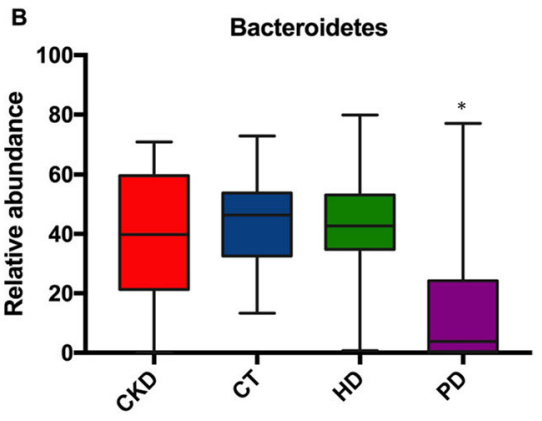

D

Proteobacteria

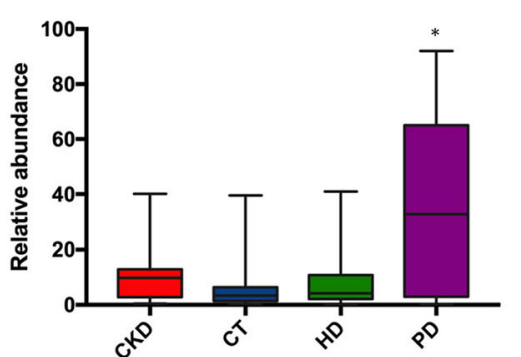

Fig. 3 Profiling histogram at the phylum classification level. a. Intestinal flora phyla were mostly abundance in Firmicutes, Bacteroidetes, Proteobacteria, Actinobacteria, and Verrucomicrobia. b. Bacteroides-dominant gut type was significantly decreased in PD group. c. Firmicutesdominant type was significantly decreased in PD group. d. Proteobacteria-dominant gut type was significantly increased in PD group 


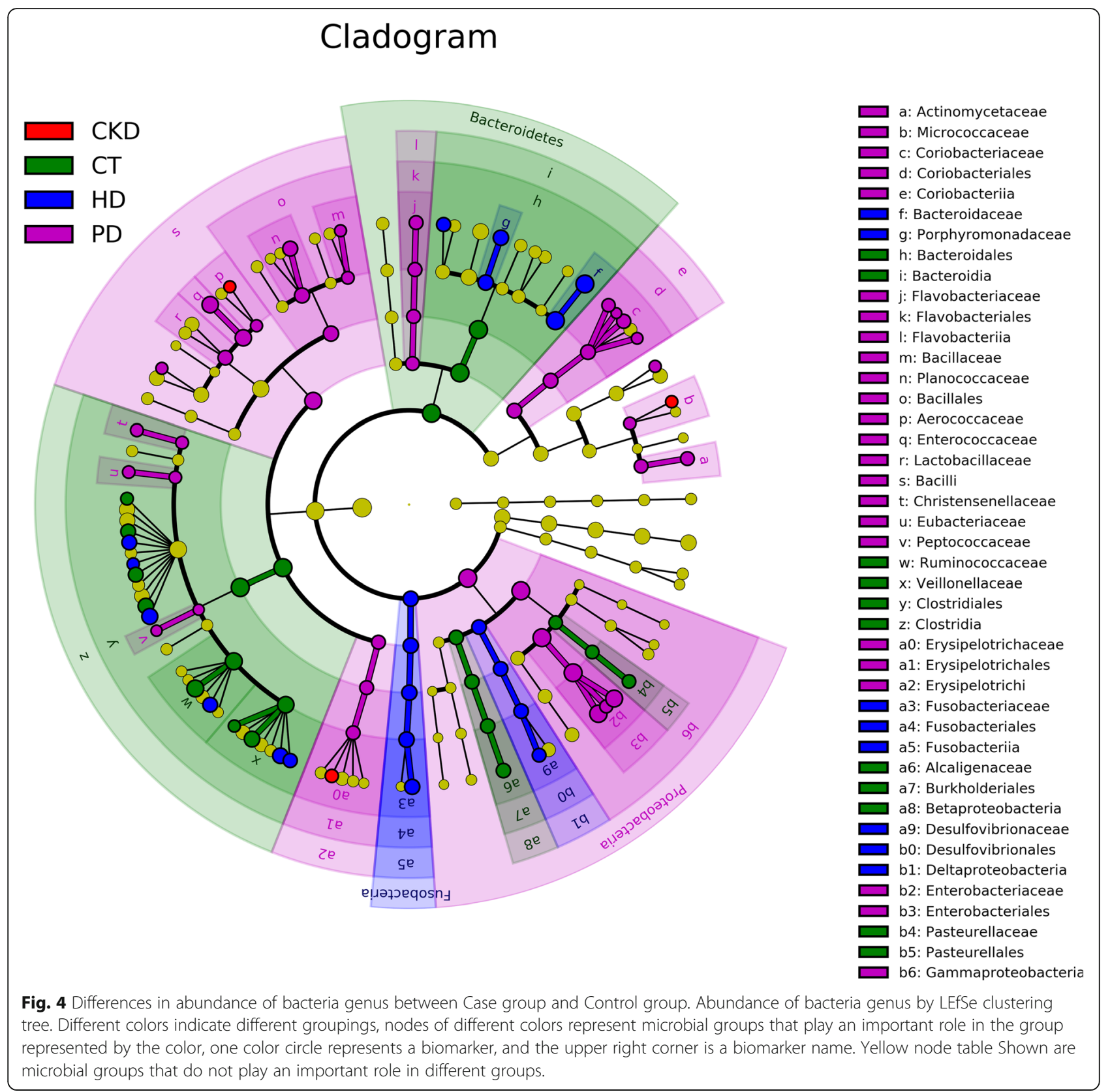

\section{Analysis of intestinal microbial function}

High-abundance bacteria KEGG level 1 pathways in the four groups were significantly enriched in metabolism, genetic information processing, and environmental information processing. The following KEGG level 2 pathways were significantly enriched in carbohydrate metabolism, amino acid metabolism, energy metabolism, translation, and membrane transport. Number of differential expressed genes in KEGG enrichment pathways: starch and sucrose metabolism, alanine aspartate and glutamate metabolism, arginine and proline metabolism, oxidative phosphorylation, ribosome, aminoacyl tRNA biosynthesis, and $\mathrm{ABC}$ transporters were significantly different in PD group compared with CT, CKD, and HD groups (Fig. 7 \& Additional file 1: Table S1).

\section{Discussion}

Gut microbiota affect physiological functions in CKD patients by modulating genes involved in host immunity, cell proliferation, and metabolism [17-19]. The pattern of renal replacement therapy also appears to influence gut microbiota [20]. Our study showed that decreased diversity and altered communities of intestinal flora in PD patients, in which microbial diversity was positive correlated with the 


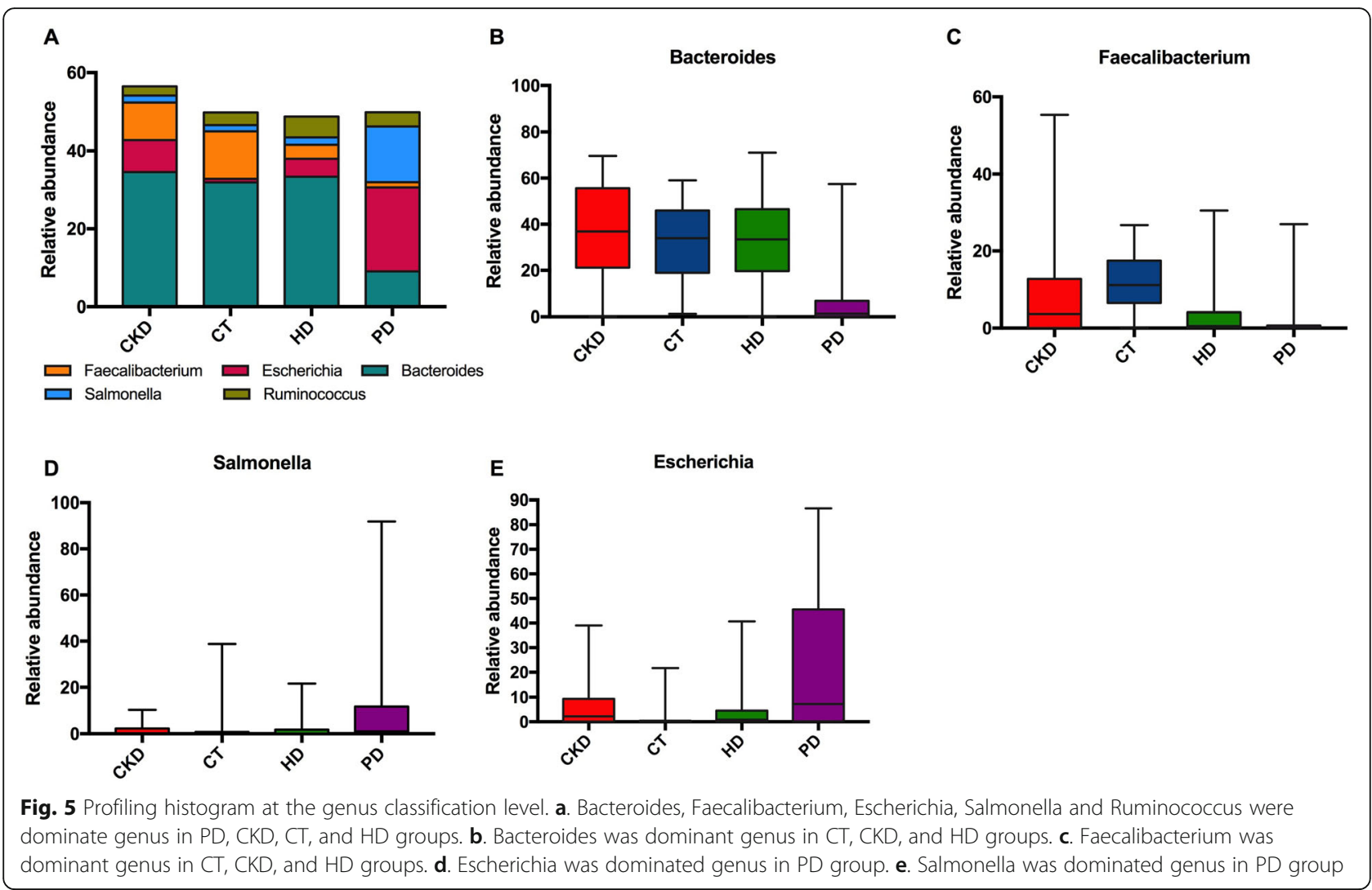

albumin level. A total of 20 intestinal flora phyla were detected in 166 fecal samples, divided into 3 dominant intestinal types including Bacteroides-dominant gut type, Firmicutes-dominant type and Proteobacteria-dominant gut type. Further analyses found 198 genera, the abundance of 86 genera were significantly different. Butyrateproducing taxa as Faecalibacterium in genera level and Bifidobacteriaceae and Prevotellaceae in family level were dominant genus in CT, CKD, and HD groups, while Urease containing-, indole- and p-cresol-forming taxa as Escherichia in genera and Enterobacteriaceae, Enterococcaceae in family level was dominated genus in PD group. Number of differential expressed genes in KEGG enrichment pathways were significantly different in PD group in carbohydrate metabolism, amino acid metabolism, energy metabolism, translation, and membrane transport.

Qualitative and quantitative changes in host microbiome profile and disruption in gut barrier resulting in gut dysbiosis was commonly seen among CKD patients [21]. A Chinese study observed no significant differences in intestinal flora diversity between CKD patients and healthy control groups, suggesting that bacterial diversity was not seriously damaged in this population [14]. Microbiota dysbiosis, which was differed between modes of dialysis, was considered a main risk factor in promoting chronic systemic inflammation in CKD patients [22]. Besides dialysis modes, age and dialysis vintage also contributed to the microbiome diversity [20]. Another Chinese study found that probiotic bacteria was less frequently detected in PD patients, which may impair host intestinal barrier and increase the risk of enteric organism invasion [23]. Diabetic patients could develop impaired renal function and induce diabetic associated cardiovascular disease [24, 25]. These diabetic cardiomyopathy patients may have abnormal bacterial metabolism [26]. Our study has further confirmed that different dialysis modes were critical contributors to microbiota alterations considered that diabetic patients have been excluded and no cardiac dysfunction have been found in our patients.

High-throughput sequencing in our study found that the intestinal flora diversity of PD patients was lower than that of $\mathrm{HD}$ and non-dialysis CKD patients, suggested that intestinal flora was seriously damaged by PD as a renal replacement therapy. Other investigation further revealed that alpha diversity was closely related to the patient's inflammatory condition $[14,20]$. Significant relationship between diversity and inflammatory factors was not found in our study, however, we revealed that patients who have higher albumin level showed with more abundance intestinal flora. This result suggested that people with better alpha diversity of the flora could have better nutrition. Improve the diversity of bacteria could be an effective way to improve the malnutrition status of dialysis patients. 


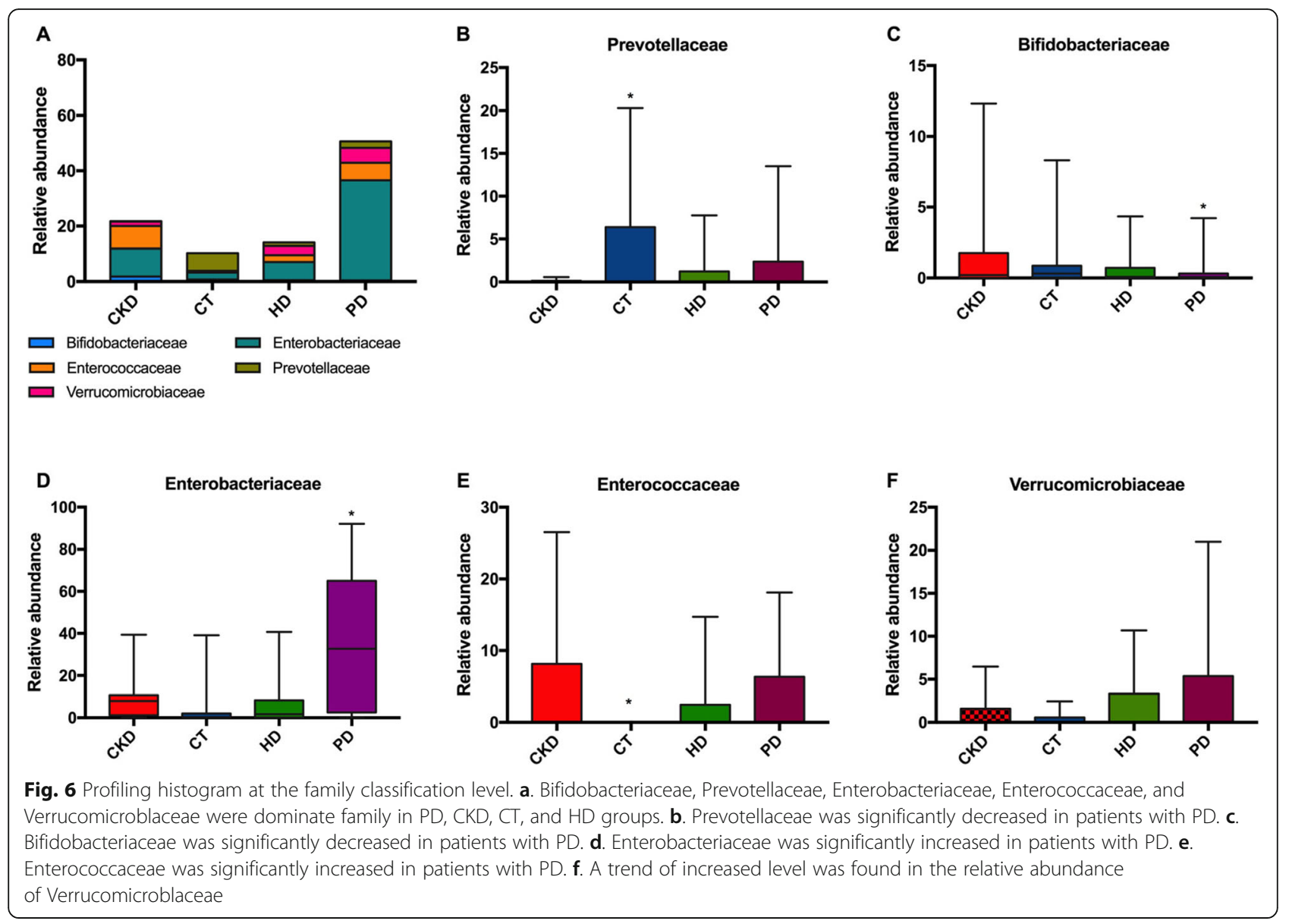

Colonic bacteria ferment indigestible carbohydrates and proteins and form short chain fatty acids (SCFAs), so that distal guts can absorb more rapidly. SCFAs were also used as cross-feeding nutrients for microorganisms, which were unable to digest macromolecules $[27,28]$ SCFAs can bind to G-protein-coupled receptors GPR41 and GPR43 for regulating inflammation in adipose tissue, intestinal cells, and immune cells $[29,30]$. A large number of homologous peptides between human and intestinal bacteria have an autoimmunogenicity effect by binding to HLA-II alleles. Most of the bacteria containing autoimmune peptides were belonged to either the phylum Firmicutes or Proteobacteria [31]. Our study showed the abundance of Proteobacteria was higher while Bacteroidetes and Firmicutes were lower in the PD group. This result suggested that the decreased Proteobacteria in patients undergoing PD might aggravate the disease situation. Further analyses on genus level revealed that compared with the other three groups, Bacteroides, Escherichia and Faecalibacterium was the dominant intestinal type in the PD group. Escherichia produce indoles which mainly affect the cardiovascular system and kidney functions and Faecalibacterium is one of the most abundant human fecal bacterial populations that produce butyrate, suggesting that
PD treatment may increase toxins released in CKD patients $[32,33]$. With the accumulation of uremic toxins and activation of inflammatory reactions in CKD patients, there is a decrease in qualitative and quantitative properties of probiotics, which in turn promotes CKD progress [34, 35]. Changed family population of Bifidobacteriaceae, Prevotellaceae and Lactobacillaceae can result in reduced butyrate level and lead to insufficient SCFAs, while families possessed Tryptophanase, indole and p-cresol-forming enzymes including Clostridiacease, Enterobacteriaceae and Verrucomicrobiaceae [36]. In our study, decreased SCFA secretion induced by decreased Bifidobacteriaceae and Prevotellaceae, increased toxins secretion induced by Enterobacteriaceae and Enterococcaceae indicated that changed population of intestinal flora may have an adverse effect on CKD patients with PD.

Gut microbiota can also affect immune system stimulation, intestinal epithelial homeostasis, vitamins B and $\mathrm{K}$ synthesis, gastrointestinal motility and function enhancement, nutrients absorption, drugs metabolism and SCFAs and polyamines production [4]. In our study, similar CKD and HD intestinal flora was correlated with similar expression of CRP and IL-6. However, significantly decreased diversity and altered communities of intestinal flora between 


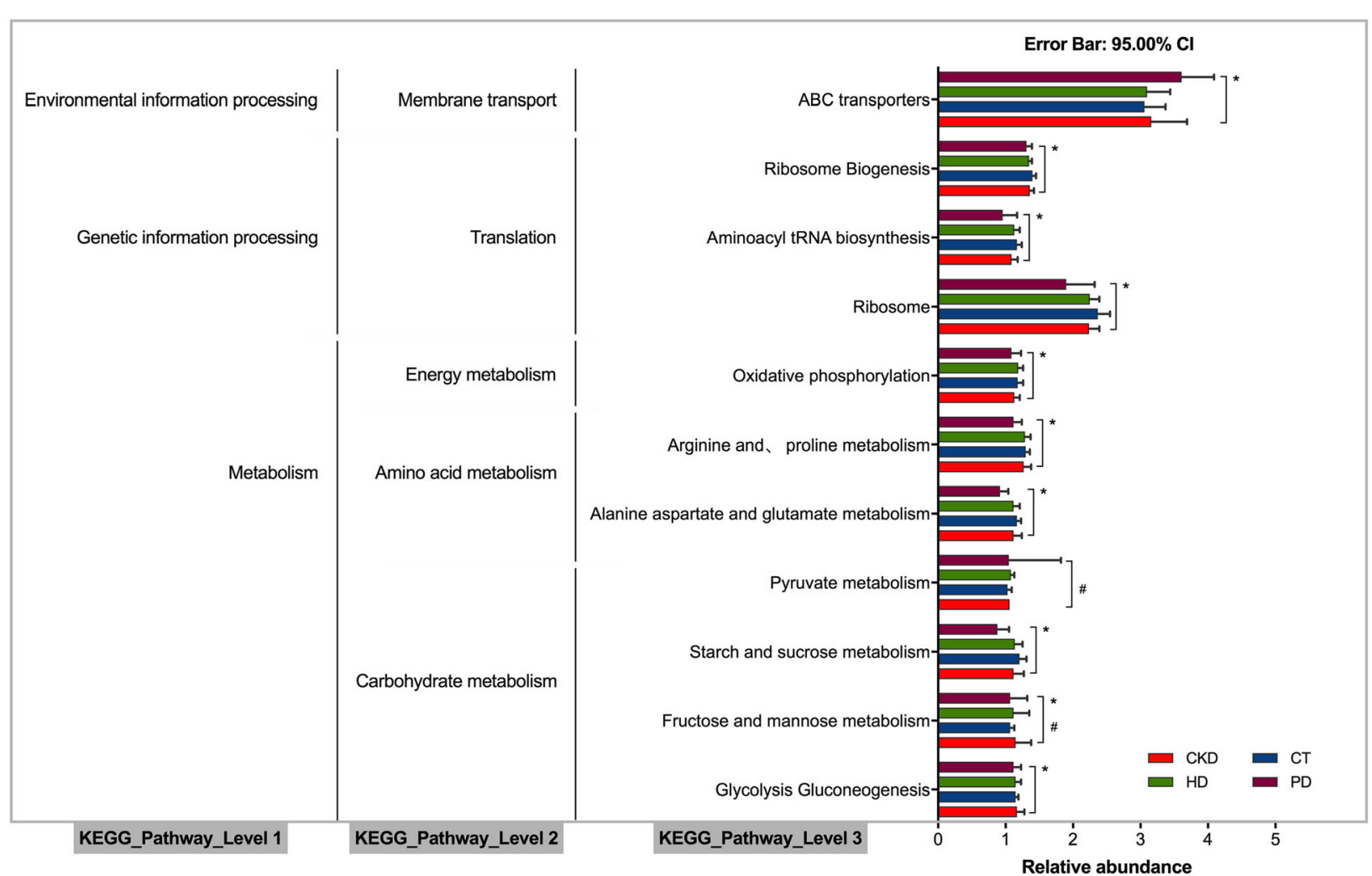

Fig. 7 KEGG pathway of abundance changed intestinal flora. Three levels of KEGG enrichment pathways in CT, CKD, HD and PD group. Highabundance bacteria KEGG level 1 pathways were significantly enriched in metabolism, genetic information processing, and environmental information processing. The KEGG level 2 pathways were significantly enriched in carbohydrate metabolism, amino acid metabolism, energy metabolism, translation, and membrane transport. The KEGG level 3 pathways were significantly enriched in starch and sucrose metabolism, alanine aspartate and glutamate metabolism, arginine and proline metabolism, oxidative phosphorylation, ribosome, aminoacyl tRNA biosynthesis, and $A B C$ transporters.

PD and HD was found correlated with excessive expression of CRP and IL-6. Butyrate-producing taxa as Faecalibacterium in genera level and Bifidobacteriaceae and Prevotellaceae in family level were dominant genus in CT, CKD, and HD groups, while urease containing-, indole- and p-cresolforming taxa as Escherichia in genera and Enterobacteriaceae, Enterococcaceae in family level was dominated genus in PD group. Our result indicated that beneficial and harmful bacteria was imbalanced in PD patients, which was more likely to induce inflammatory response of ESRD patients. CRP and IL-6 as inflammatory markers need further analysis. In this study, functional analysis of the microbiome with altered expression in CKD patients under different therapeutic states suggests that changes in the abundance of certain microbial species in plays an important role in affecting metabolic functionality and inflammatory response. Notably, a regulatory effect on bacterial chemotaxis was found in level 3 KEGG analysis. Bacteria moved under the control of a complex signal transduction system, moving toward the production of beneficial chemicals or away from unfavorable chemicals. The signal of bacterial chemotaxis may be the mechanism of escaping the antibacterial effect of antibiotics and aggravating the disease, although future investigation is required to confirm this.

The present study has certain limitations. First, this was a single-center research, the generalizability of altered microbiota in CKD patients with different hemodialysis therapy is limited. Second, the lack of animal model experiments to validate the effect of improved microbial diversity to patient's malnutrition status was also a limitation in our study. Further CKD animal models were needed in verify to verify the possible role of intestinal flora in recovery of CKD patients. In spite of these limitations, our results provide information regarding the altered microbial diversity and communities in Chinese CKD patients with different hemodialysis therapy and suggest that we should raise attention on intestinal flora of CKD patients with PD therapy. The influence of different primary renal diseases on microbiota alteration needs further investigation. 


\section{Conclusion}

In conclusion, peritoneal dialysis therapy could result in reduced diversity and altered microbial communities, with reduced probiotic butyrate-producing taxa and increased urease containing-, indole- and p-cresol-forming taxa. The disordered intestinal flora can seriously affect the nutrition level in CKD patients with PD therapy.

\section{Supplementary information}

Supplementary information accompanies this paper at https://doi.org/10. 1186/s12882-019-1668-4.

Additional file 1 Table S1. KEGG level 3 pathways of abundance changed intestinal flora.

\section{Abbreviations}

CKD: Chronic kidney disease; CT: Control; HD: Hemodialysis; KEGG: Kyoto Encyclopedia of Genes and Genomes; PD: Peritoneal dialysis; SCFAs: Shortchain fatty acids; TMAO: Trimethylamine $\mathrm{N}$-oxide

\section{Acknowledgements}

We thank Beijing Genomics Institute for technical support in this project.

\section{Authors' contributions}

JGH designed the study protocol, analyzed data and wrote the main manuscript, $X S Z, J Y$ and DYZ collecting data, DPQ, XX and $Y Y Z$ did the statistical analysis, YL was a guarantor of integrity of the entire study. All authors read and approved the final manuscript.

\section{Funding}

This work was supported by Science and Technology Planning Project of Guangdong Province of China (Grant No. 2016A020215023) in the study design and data collection, and Science and Technology Planning Project of Guangdong Province of China (Grant No. 2017B090904027) in data analysis and manuscript written.

\section{Availability of data and materials}

All data generated or analyzed during this study are included in this published article.

\section{Ethics approval and consent to participate}

The written informed consent was signed by all patients. This study was approved by ethics committee of the Guangzhou Red Cross Hospital [No. 2017-032-01/02]. The study adhered to the tenets of the Declaration of Helsinki and the Guidance on Sample Collection of Human Genetic Diseases by the Ministry of Public Health of China.

\section{Consent for publication}

We demonstrated that our manuscript have followed the principle of anonymity, no direct or indirect identifiers of our participants were used for publication.

\section{Competing interests}

The authors declare that they have no competing interests.

Received: 28 September 2019 Accepted: 19 December 2019

Published online: 13 January 2020

\section{References}

1. Blum HE. The human microbiome. Adv Med Sci. 2017;62(2):414-20.

2. Human Microbiome Project C. Structure, function and diversity of the healthy human microbiome. Nature. 2012;486:207-14. https://doi.org/10. 1038/nature11234.

3. Faith JJ, Guruge JL, Charbonneau M, Subramanian S, Seedorf H, Goodman $\mathrm{AL}$, et al. The long-term stability of the human gut microbiota. Science. 2013;341:1237439. https://doi.org/10.1126/science.1237439.
4. Dominguez-Bello MG, Blaser M, Ley RE, Knight R. Development of the human gastrointestinal microbiota and insights from high-throughput sequencing. Gastroenterology. 2011;140:1713-9. https://doi.org/10.1053/j.gastro.2011.02.011.

5. Carding S, Verbeke K, Vipond DT, Corfe BM, Owen $\amalg$. Dysbiosis of the gut microbiota in disease. Microb Ecol Health Dis. 2015;26:26191. https://doi.org/ 10.3402/mehd.v26.26191.

6. Jose PA, Raj D. Gut microbiota in hypertension. Curr Opin Nephrol Hypertens. 2015;24:403-9. https://doi.org/10.1097/MNH.0000000000000149.

7. Natarajan N, Pluznick JL. From microbe to man: the role of microbial short chain fatty acid metabolites in host cell biology. Am J Phys Cell Phys. 2014; 307:C979-85. https://doi.org/10.1152/ajpcell.00228.2014.

8. Han JL, Lin HL. Intestinal microbiota and type 2 diabetes: from mechanism insights to therapeutic perspective. World J Gastroenterol. 2014;20:1773745. https://doi.org/10.3748/wjg.v20.147.17737

9. Festi D, Schiumerini R, Eusebi LH, Marasco G, Taddia M, Colecchia A. Gut microbiota and metabolic syndrome. World J Gastroenterol. 2014;20:1607994. https://doi.org/10.3748/wjg.v20.143.16079.

10. Qin N, Yang F, Li A, Prifti E, Chen Y, Shao L, et al. Alterations of the human gut microbiome in liver cirrhosis. Nature. 2014;513:59-64. https://doi.org/10. 1038/nature13568.

11. Anders HJ, Andersen $\mathrm{K}$, Stecher B. The intestinal microbiota, a leaky gut, and abnormal immunity in kidney disease. Kidney Int. 2013;83:1010-6. https:// doi.org/10.1038/ki.2012.440.

12. Vaziri ND, Wong J, Pahl M, Piceno YM, Yuan J, DeSantis TZ, et al. Chronic kidney disease alters intestinal microbial flora. Kidney Int. 2013;83:308-15. https://doi.org/10.1038/ki.2012.345.

13. Pluznick JL. Gut microbiota in renal physiology: focus on short-chain fatty acids and their receptors. Kidney Int. 2016;90:1191-8. https://doi.org/10. 1016/j.kint.2016.06.033

14. Jiang $S$, Xie S, LV D, Wang $P, H e ~ H$, Zhang $T$, et al. Alteration of the gut microbiota in Chinese population with chronic kidney disease. Sci Rep. 2017:7:2870. https://doi.org/10.1038/s41598-017-02989-2.

15. Andersen K, Kesper MS, Marschner JA, Konrad L, Ryu M, Kumar Vr S, et al. Intestinal Dysbiosis, barrier dysfunction, and bacterial translocation account for CKD-related systemic inflammation. J Am Soc Nephrol. 2017;28:76-83. https://doi.org/10.1681/ASN.2015111285.

16. Mclntyre CW, Harrison LE, Eldehni MT, Jefferies HJ, Szeto CC, John SG, et al. Circulating endotoxemia: a novel factor in systemic inflammation and cardiovascular disease in chronic kidney disease. Clin J Am Soc Nephrol. $2011 ; 6: 133-41$. https://doi.org/10.2215/CJN.04610510.

17. Min YW, Rhee PL. The role of microbiota on the gut immunology. Clin Ther. 2015:37:968-75. https://doi.org/10.1016/j.clinthera.2015.03.009.

18. Hooper LV, Midtvedt T, Gordon Jl. How host-microbial interactions shape the nutrient environment of the mammalian intestine. Annu Rev Nutr. 2002; 22:283-307. https://doi.org/10.1146/annurev.nutr.22.011602.092259.

19. Backhed F, Ley RE, Sonnenburg JL, Peterson DA, Gordon Jl. Host-bacterial mutualism in the human intestine. Science. 2005:307:1915-20. https://doi. org/10.1126/science.1104816

20. Stadlbauer V, Horvath A, Ribitsch W, Schmerbock B, Schilcher G, Lemesch S, et al. Structural and functional differences in gut microbiome composition in patients undergoing haemodialysis or peritoneal dialysis. Sci Rep. 2017;7:15601. https:/doi. org/10.1038/s41598-017-15650-9.

21. Nallu A, Sharma S, Ramezani A, Muralidharan J, Raj D. Gut microbiome in chronic kidney disease: challenges and opportunities. Transl Res. 2017;179: 24-37. https://doi.org/10.1016/j.trsl.2016.04.007.

22. Li FX, Wang MH, Wang JP, Li RS, Zhang YQ. Alterations to the gut microbiota and their correlation with inflammatory factors in chronic kidney disease. Front Cell Infect Microbiol. 2019;9:206

23. Wang IK, Lai HC, Yu CJ, Liang CC, Chang CT, Kuo HL, et al. Real-time PCR analysis of the intestinal microbiotas in peritoneal dialysis patients. Appl Environ Microbiol. 2012;78:1107-12

24. Doshi SM, Friedman AN. Diagnosis and management of type 2 diabetic kidney disease. Clin J Am Soc Nephrol. 2017;12:1366-73.

25. Go AS, Chertow GM, Fan D, McCulloch CE, Hsu CY. Chronic kidney disease and the risks of death, cardiovascular events, and hospitalization. N Engl J Med. 2004:351:1296-305.

26. Marie Bastin FA. The gut microbiota and diabetic cardiomyopathy in humans. Diabetes Metab. 2019; https://doi.org/10.1016/j.diabet.2019.10.003.

27. Rios-Covian D, Ruas-Madiedo P, Margolles A, Gueimonde M, de Los Reyes-Gavilan CG, Salazar N. Intestinal short chain fatty acids and their link with diet and human health. Front Microbiol. 2016;7:185. https://doi.org/10.3389/fmicb.2016.00185. 
28. den Besten G, Lange K, Havinga R, van Dijk TH, Gerding A, van Eunen K, et al. Gut-derived short-chain fatty acids are vividly assimilated into host carbohydrates and lipids. Am J Physiol Gastrointest Liver Physiol. 2013;305: G900-10. https://doi.org/10.1152/ajpgi.00265.2013.

29. Brown AJ, Goldsworthy SM, Barnes AA, Eilert MM, Tcheang L, Daniels D, et al. The orphan G protein-coupled receptors GPR41 and GPR43 are activated by propionate and other short chain carboxylic acids. J Biol Chem. 2003;278:11312-9. https://doi.org/10.1074/jbc. M211609200.

30. Kimura I, Ozawa K, Inoue D, Imamura T, Kimura K, Maeda T, et al. The gut microbiota suppresses insulin-mediated fat accumulation via the short-chain fatty acid receptor GPR43. Nat Commun. 2013;4:1829. https://doi.org/10. 1038/ncomms 2852 .

31. Negi S, Singh H, Mukhopadhyay A. Gut bacterial peptides with autoimmunity potential as environmental trigger for late onset complex diseases: in-silico study. PLoS One. 2017;12:e0180518. https://doi.org/10. 1371/journal.pone.0180518.

32. Summers SC, Quimby JM, Isaiah A, Suchodolski JS, Lunghofer PJ, Gustafson $\mathrm{DL}$. The fecal microbiome and serum concentrations of indoxyl sulfate and p-cresol sulfate in cats with chronic kidney disease. J Vet Intern Med. 2019; 33:662-9. https://doi.org/10.1111/jvim.15389.

33. Louis P, Flint HJ. Diversity, metabolism and microbial ecology of butyrateproducing bacteria from the human large intestine. FEMS Microbiol Lett. 2009;294:1-8. https://doi.org/10.1111/j.1574-6968.2009.01514.x.

34. Mazmanian SK, Round JL, Kasper DL. A microbial symbiosis factor prevents intestinal inflammatory disease. Nature. 2008;453:620-5. https://doi.org/10. 1038/nature07008

35. Atarashi K, Tanoue T, Shima T, Imaoka A, Kuwahara T, Momose Y, et al. Induction of colonic regulatory $T$ cells by indigenous Clostridium species. Science. 2011;331:337-41. https://doi.org/10.1126/science.1198469.

36. Wong J, Piceno YM, DeSantis TZ, Pahl M, Andersen GL, Vaziri ND. Expansion of urease- and uricase-containing, indole- and p-cresol-forming and contraction of short-chain fatty acid-producing intestinal microbiota in ESRD. Am J Nephrol. 2014;39:230-7. https://doi.org/10.1159/000360010.

\section{Publisher's Note}

Springer Nature remains neutral with regard to jurisdictional claims in published maps and institutional affiliations.

Ready to submit your research? Choose BMC and benefit from:

- fast, convenient online submission

- thorough peer review by experienced researchers in your field

- rapid publication on acceptance

- support for research data, including large and complex data types

- gold Open Access which fosters wider collaboration and increased citations

- maximum visibility for your research: over $100 \mathrm{M}$ website views per year

At $\mathrm{BMC}$, research is always in progress.

Learn more biomedcentral.com/submissions 